\title{
Video Article \\ Use of a Piglet Model for the Study of Anesthetic-induced Developmental Neurotoxicity (AIDN): A Translational Neuroscience Approach
}

\author{
Emmett E. Whitaker ${ }^{1,2}$, Christopher Z. Zheng ${ }^{1}$, Bruno Bissonnette ${ }^{1,2,3}$, Andrew D. Miller ${ }^{4}$, Tanner L. Koppert ${ }^{1,2}$, Joseph D. Tobias ${ }^{1,2}$, Christopher \\ R. Pierson ${ }^{5,6}$, Fedias L. Christofi ${ }^{1}$ \\ ${ }^{1}$ Department of Anesthesiology, Ohio State University College of Medicine \\ ${ }^{2}$ Department of Anesthesiology and Pain Medicine, Nationwide Children's Hospital \\ ${ }^{3}$ Department of Anaesthesia and Critical Care Medicine, University of Toronto \\ ${ }^{4}$ Department of Biomedical Sciences, Section of Anatomic Pathology, Cornell University College of Veterinary Medicine \\ ${ }^{5}$ Department of Pathology and Anatomy, Ohio State University College of Medicine \\ ${ }^{6}$ Department of Pathology and Laboratory Medicine, Nationwide Children's Hospital
}

Correspondence to: Emmett E. Whitaker at emmett.whitaker@nationwidechildrens.org

URL: https://www.jove.com/video/55193

DOI: doi:10.3791/55193

Keywords: Medicine, Issue 124, Piglets, neurotoxicity, anesthesia, neuroinflammation, neurocognitive outcome, neurodevelopment, isoflurane, hippocampus

Date Published: 6/11/2017

Citation: Whitaker, E.E., Zheng, C.Z., Bissonnette, B., Miller, A.D., Koppert, T.L., Tobias, J.D., Pierson, C.R., Christofi, F.L. Use of a Piglet Model for the Study of Anesthetic-induced Developmental Neurotoxicity (AIDN): A Translational Neuroscience Approach. J. Vis. Exp. (124), e55193, doi:10.3791/55193 (2017).

\section{Abstract}

Anesthesia cannot be avoided in many cases when surgery is required, particularly in children. Recent investigations in animals have raised concerns that anesthesia exposure may lead to neuronal apoptosis, known as anesthesia-induced developmental neurotoxicity (AIDN). Furthermore, some clinical studies in children have suggested that anesthesia exposure may lead to neurodevelopmental deficits later in life. Nonetheless, an ideal animal model for preclinical study has yet to be developed. The neonatal piglet represents a valuable model for preclinical study, as they share a striking number of developmental similarities with humans.

The anatomy and physiology of piglets allow for implementation of rigorous human perioperative conditions in both survival and non-survival procedures. Femoral artery catheterization allows for close monitoring, thus enabling prompt correction of any deviation of the piglet's vital signs and chemistries. In addition, there are multiple developmental similarities between piglets and human neonates. The techniques required to use piglets for experimentation will require experience to master. A pediatric anesthesiologist is a critical member of the investigative team. We describe, in a general sense, the appropriate use of a piglet model for neurodevelopmental study.

\section{Video Link}

The video component of this article can be found at https://www.jove.com/video/55193/

\section{Introduction}

Each year, millions of children in the USA receive general anesthesia, many of them under 4 years of age ${ }^{1}$. Anesthetic-induced developmental neurotoxicity (AIDN) has become a focus of pediatric anesthesia research as it has become imperative to gain an understanding the effects of anesthesia on immature brains. Previous research has shown that commonly used anesthetics, such as isoflurane, can cause increased neuronal apoptosis in the brains of young animals. Studies in children have yielded equivocal results ${ }^{2}$. Understanding the pathogenesis of AIDN, identifying potential therapeutic targets for its prevention or amelioration, and describing the safest anesthetic regimens available have become urgent goals of the pediatric anesthesia community. The primary aim of this study was to develop an optimal animal model and method for quantifying the effects of anesthetics on the developing brain, and to stimulate carefully designed investigation into the safety of currently widely used anesthetics.

In a recent systematic review of the current body of preclinical literature on AIDN, the authors noted significant methodological heterogeneity in over 900 studies $^{3}$. Many considered this to be a call for a clinically relevant, well-designed preclinical model, which does not yet exist despite several years of research on this subject. Most rodent models, out of necessity, use an approach that does not allow rigorous physiologic monitoring, blood sampling, or mechanical ventilation. Since the brain is exquisitely sensitive to physiologic derangements, it is difficult to rely upon the results from such models. A primary goal for the development of this model was to design it in such a way that all physiologic variables such as blood gas parameters, body temperature, respiratory parameters etc. are monitored and corrected when necessary.

Additionally, because the neurotoxicity of anesthesia likely depends on the developmental progress of the brain at the time of exposure, an animal model should be chosen that can best mimic the development and structure of the neonatal human brain, to maximize the translational 
relevance of the results ${ }^{4}$. The translational piglet model provides the level of clinical relevance sought after in these reviews and editorials, as it is designed to address this need for pertinent preclinical data that can inform future clinical studies.

Isoflurane, a GABA type $A\left(G_{A B A}\right)$ receptor agonist and weak NMDA receptor antagonist, is the is a commonly used inhaled anesthetic in clinical practice worldwide. Anesthetics like isoflurane have been considered safe so long as they do not induce hypotension or hypoxia; yet, more subtle effects may be occurring. When the brain is exposed to general anesthesia, the balance of GABA agonism and NMDA antagonism is disrupted, resulting in alterations in cellular architecture, connectivity, and function. In addition, while GABA is generally an inhibitory neurotransmitter, it is known to be excitatory in immature brains ${ }^{5}$. Precisely when GABA's transition from excitatory to inhibitory occurs is not well understood, and is likely species-dependent.

When an imbalance between excitatory and inhibitory input in the brain occurs during the so-called "brain growth spurt", the resulting excitotoxic dysregulation of critical molecular pathways may lead to abnormal neurodevelopment, such as apoptotic neurodegeneration. In addition to increased apoptosis, oxidative stress and inflammation may also be induced, while neuronal cell proliferation, neuronal migration, and axonal arborization become suppressed or dysregulated ${ }^{6}$. The net result is neurocognitive disturbances that may persist into adulthood ${ }^{2}$.

To directly measure the neurotoxic effects of isoflurane on young mammals, neonatal piglets are used. Piglets share more CNS similarities with humans than any other mammal, and as such, their neurodevelopmental and neuroanatomical similarities make them an ideal animal for a clinically-relevant mammalian model of AIDN. Both humans and piglets possess gyrencephalic brains, sharing similarities in the character and distribution of brain gyri, gray matter, and white matter. The piglet hippocampus, basal ganglia, and brainstem are also topographically similar to that of humans ${ }^{7}$. Developmentally, piglets are one of the few non-human mammals that undergo perinatal brain growth and myelination ${ }^{8}$. In utero, both human and piglet brains undergo significant growth during late trimester gestation. In correlation, at birth, human and piglet brains are $27 \%$ and $25 \%$ of adult brains, respectively. Magnetic resonance imaging has revealed that a one week old piglet brain is approximately equivalent to a one month old human brain in terms of neuronal maturation and dendritic arborization ${ }^{9}$. Additionally, the piglet and human brain share numerous similarities with respect to patterns of neurodevelopment. For example, the expression and mRNA sequence of reelin ${ }^{10}$, the topographical distribution of $5-\mathrm{HT}$ neurons ${ }^{11}$, and neural tube closure ${ }^{12}$ are all parallel to that which is seen in humans. Furthermore, there is extensive homology between the genomes of piglets and humans ${ }^{13}$

The relevance of an animal model must be understood in the context of human pathology, particularly in relation to the brain maturity and pathobiology of the human infant. Most of the existing anesthesia toxicity studies use a rodent model, with a few utilizing non-human primate models. However, rodents and primates may not be the ideal animals for investigating AIDN.

Though widely used, rodent brains are very different from that of humans throughout development. Most notably, rodents possess lissencephalic (or smooth) brains. The rodent brain lacks the gyri and sulci that are characteristic of more neurologically complex organisms. The rodent brain also undergoes a postnatal brain growth spurt ${ }^{14}$, dissimilar to humans and piglets. It has been observed that there are variations in the vulnerability of different brain regions to inhaled anesthetic ${ }^{15}$. Therefore, it should be important that the animal model for studying AIDN possess a brain that is neurodevelopmentally and neuroanatomically similar to that of a human, so as to best model the anesthesia-induced brain alterations that is likely to be seen in pediatric patients. As described previously, piglets possess a brain that is much better suited for this role. Furthermore, the common forms of rodent neurocognitive testing, such as spatial learning and memory evaluated in the Morris water maze, are not directly relevant or comparable to the neurocognitive assessments in young children ${ }^{16}$. One of the advantages of using piglets for developmental neuroscience is that they are extremely amenable to neurocognitive testing, even at an early age. Numerous neurocognitive tests that are considered useful for other mammalian species have been successfully used and validated in pigs. While still an evolving field, neurocognitive assessment in piglets includes more complex tests that better mimics human deficits, such as an inclined beam motor test ${ }^{17,18}$ and mirror spatial awareness test ${ }^{19}$. Motor testing with the inclined beam, as part of traumatic brain injury study in piglets, shows high reliability in assessment of motor function. The mirror test demonstrates memory of the surrounding environment plus recognition and utilization of a reflected image to seek out food reward.

On the other hand, non-human primates may be a more appropriate model for pediatric anesthesia studies, but there are a number of prohibitive factors, including cost and difficulty of use. Additionally, they are extremely sensitive to early rearing conditions, particularly stress and maternal separation ${ }^{20}$. Factors important to the study of AIDN, such as allosteric modulators, receptor-ligand affinities, post-translational modifications, receptor subunit compositions, and alternative splicing variants, are unknown in the case of primates. This is because genes relevant to such concepts have not been cloned. In contrast, they have been cloned in pigs. As such, only limited work has been done in non-human primates $^{21,22}$.

The piglet model capitalizes on the advantages of the rodent and non-human primate models: it is cost effective, easy to use relative to nonhuman primate studies, and is neuroanatomically and neurophysiologically similar to the pediatric human brain. The use of piglet in neuroscience research has grown in the recent years, including a number of studies that have examined pediatric neuroinflammatory conditions. Effects of respiratory viral infection on hippocampus and spatial learning ${ }^{23}$, reducing brain cell death following stroke ${ }^{24}$, neurogenesis following traumatic brain injury ${ }^{25}$, and enzyme activity during seizures ${ }^{26}$ are some of the studies that have used neonatal piglets. This substantial and growing body of literature lends strength to the suitability and sustainability of the clinically relevant and highly reproducible piglet model for the study of anesthesia-induced neurotoxicity.

\section{Protocol}

Healthy, domestic piglets (Sus scrofa) are obtained from a farm approved by The Ohio State University Institutional Animal Care and Use Committee (IACUC). All animal experimentations are performed in accordance with The Ohio State University IACUC policy, after protocol approval. 


\section{Animals and Animal Handling}

1. Use male piglets in this experiment to eliminate the potential confounding effects of sex. NOTE: If experimental goals include evaluation of effects of the experiment on animals while in the period of maximal brain growth, do not use piglets older than 14 days.

2. Schedule the piglets to arrive in the vivarium at least $24 \mathrm{~h}$ before experimentation to allow acclimation to the environment. NOTE: Trained veterinary technicians supervised by licensed veterinarians provide routine animal care.

1. Keep the piglets in individual temperature-controlled cages and give a nutritionally complete, commercial piglet milk replacer ad libitum Supply the animals with a blanket and a toy. Continuously monitor the temperature in the animal enclosures.

3. For this preliminary feasibility study, used 18 piglets for the isoflurane arm and 22 piglets for the control arm. Perform sample size calculations based on study design when possible. Randomize the available piglets to either control or exposure group for the appropriate length of exposure. From experience, even with multiple investigators, expect to be able to perform no more than 2 experiments per day ( 2 animals total).

\section{Control Animals}

1. Do not perform experimental intervention on control animals.

2. Induce deep general anesthesia via face cone mask for the perfusion and tissue collection procedure. Specifically, after the 24-h acclimation period, anesthetize the piglets with $5 \%$ isoflurane or $8 \%$ sevoflurane in $100 \%$ oxygen via face cone mask. Do not use desflurane for induction.

NOTE: The time between induction of anesthesia and institution of cold PBS perfusion should be as short as possible. Experienced technicians can complete this process in under 5 min.

1. Confirm adequate depth of anesthesia by lack of dewclaw-pinch reflex using a surgical clamp.

2. To avoid hypoxic/ischemic insults to the brain, monitor the piglet using a pulse oximeter to ensure maintenance of adequate oxygenation, ventilation, and cardiac output until perfusion of cold phosphate-buffered saline (PBS) begins.

NOTE: To provide additional protection against tissue damage, pack the animal (including the head) in ice after induction of anesthesia.

3. Perform a transcardiac perfusion.

NOTE: because paraformaldehyde is used, the perfusion procedure should be performed under a fume hood or on a downdraft table.

1. Make a craniocaudal incision along the length of the sternum using a scalpel. The depth of the incision should be sufficient to expose the sternum.

2. Carefully, perform a midline sternotomy with a pair of sharp heavy scissors, avoiding damage to the heart, lungs, or diaphragm. If necessary, place a finger placed between the posterior aspect of the sternum and the intrathoracic contents to avoid injuries. Maneuver a finger into the mediastinum by making a small (finger-sized) incision in the diaphragm.

3. After entering the thoracic cavity, keep the rib cage open using a self-retaining retractor.

4. Incise the pericardium using forceps and a pair of scissors, exposing the beating heart. Use caution not to injure the heart.

5. Identify the left ventricle and carefully place a cannula (such as a $14 \mathrm{G}$ angiocatheter) through the apex of the ventricle. Remove the needle, leaving the catheter in place.

NOTE: Use caution not to puncture the posterior wall of the ventricle. Pulsatile blood return from the catheter indicates that it is properly placed. Blood can easily be sampled from the animal at this point.

6. After identifying the right atrium, perform an atriotomy by making a large incision in the atrium with scissors to allow for exsanguination and the escape of perfusate.

NOTE: Isoflurane via inhalation should be continued until cardiac death is confirmed. Cardiac death is confirmed by direct observed lack of cardiac output.

4. Perfuse the piglet using a perfusate consisting of cold $\left(4^{\circ} \mathrm{C}\right)$ phosphate-buffered saline (PBS) containing heparin at a concentration of 5 units per $\mathrm{mL}$. Perfuse at $\mathbf{3 0 0} \mathrm{mL}$ per $\min$ for $5 \mathrm{~min}$ or until the solution runs clear.

1. Use caution that the perfusion cannula does not become dislodged during perfusion. Use a commercially available peristaltic pump for this and all other perfusions.

5. Perform a hemicraniectomy to remove one hemisphere of the brain for fresh tissue analysis.

NOTE: This protocol allows for retrieval of one hemisphere of fresh brain tissue. The other hemisphere is fixed. If no fresh tissue is required, skip to step 2.6.

1. During this procedure, continue the circulation of ice-cold PBS at a rate of $50 \mathrm{~mL}$ per hour to ensure that the brain remains cold.

2. Make a longitudinal incision in the scalp along the length of the sagittal suture until the foramen magnum using a scalpel. During the process, use firm pressure to create a score in the skull. Reflect the scalp to expose the entire cranium.

3. Using rongeurs and beginning at the foramen magnum, remove the skull on one side by inserting the rongeurs between the skull and the dura mater, using caution not to injure the underlying brain tissue. Remove bone in pieces, using the rongeurs to pry it away from the brain parenchyma.

4. Once the skull has been removed, incise and remove the dura mater using forceps and scissors, again using caution not to injure the underlying brain tissue.

5. Place a scalpel blade between the two hemispheres to carefully divide the corpus callosum.

6. Using a flat tool such as the broad handle end of forceps, gently retract the frontal lobe, gradually severing the cranial nerves, working anterior to posterior. At the most posterior aspect of the hemisphere, use a scalpel to cut the spinal cord. Remove the hemisphere en bloc.

NOTE: Unfixed brain tissue is fragile. Use caution when removing the hemisphere to prevent disruption of the remaining hemisphere's blood supply.

7. Section the removed hemisphere. If indicated, immediately flash-freeze in 2 -methylbutane cooled to $-160{ }^{\circ} \mathrm{C}$ in a liquid nitrogen bath to avoid tissue breakdown, and immediately stored at $-80^{\circ} \mathrm{C}$ for later analysis. 
NOTE: We recommend sectioning brain coronally in $2 \mathrm{~mm}$ increments using a matrix, but specific details of sectioning will depend upon specific experimental goals.

6. Change the perfusate to $4 \%$ paraformaldehyde (PFA). Continue the PFA perfusion at $300 \mathrm{~mL}$ per minute for at least $5 \mathrm{~min}$.

CAUTION! PFA is toxic, avoid contact with skin, eyes, or mucous membranes. Do not inhale PFA fumes.

7. Expect the piglet's body to stiffen due to the formation of aldehyde cross-linkages being created in muscle. After perfusion of PFA is complete, remove the remaining hemisphere in identical fashion to that described in step 2.5.5.

NOTE: Properly perfused brain will be pale and completely exsanguinated.

1. Place the remaining hemisphere in a small container with fresh $4 \%$ PFA at $4{ }^{\circ} \mathrm{C}$. Keep the hemisphere in PFA for $24-48 \mathrm{~h}$ to complete the fixation process.

2. After $24-48 \mathrm{~h}$, move the fixed brain to a solution of PBS containing $0.1 \%$ sodium azide, as it is essential to prevent over-fixation. Overfixation could result in masking of the epitope or strong non-specific background staining. The addition of sodium azide prevents bacterial growth.

NOTE: The tissue can be stored for up to one month at $4{ }^{\circ} \mathrm{C}$.

\section{Isoflurane (Experimental) Animals}

NOTE: Any anesthetic or intervention can be used, but we do not recommend desflurane for inhalation induction.

1. Induction and maintenance of anesthesia:

1. Perform the anesthesia using a clinical anesthesia workstation equipped with a pediatric ventilator and monitoring devices.

2. After the $24 \mathrm{~h}$ acclimation period, anesthetize the piglets with $8 \%$ sevoflurane in $100 \% \mathrm{O}_{2}$ via face cone mask.

3. Continuously monitor pulse oximetry, non-invasive blood pressure, electrocardiography, and temperature during the induction period and at all times during the study procedure.

4. After induction, titrate sevoflurane or isoflurane to a concentration that allows for adequate depth of anesthesia while ensuring sustained spontaneous respiration (typically at a concentration of 3-4\%).

5. Place a $24 \mathrm{G}$ peripheral intravenous catheter in the marginal ear vein (Figure 1).

6. Place the piglet in the dorsal recumbent position for tracheal intubation (Figure 2, panel A). Use a Miller \#1 or \#1.5 blade to facilitate instrumentation of the hypopharynx and intubation of the trachea. NOTE: An experienced operator and assistant are needed during laryngoscopy.

1. Have an assistant displace the tongue of the animal using a dry gauze to facilitate exposure of the larynx and visualization of the vocal cords (Figure 2B).

NOTE: The piglet epiglottis is morphologically similar to that of humans (Figure 2C). The piglet vocal cords can be difficult to visualize since they are several millimeters deep within the laryngeal inlet (Figure 2D).

2. Displace the epiglottis: place the tip of the laryngoscope blade underneath the epiglottis and lift the blade upwards to expose the larynx.

3. Prior to placement of the tube into the trachea, spray the vocal cords with $0.5 \mathrm{~mL}$ of $2 \%$ lidocaine to prevent laryngospasm during the passage of the endotracheal tube, as piglets are especially prone to laryngospasm.

7. Place and secure a $3.0 \mathrm{~mm}$ cuffed tracheal tube.

1. Ensure bilateral breath sounds and sustained end-tidal carbon dioxide using chest auscultation with a stethoscope and $\mathrm{EtCO}_{2}$ monitoring.

2. Inflate the piglet's lungs to a continuous airway pressure of $20 \mathrm{~cm} \mathrm{H} \mathrm{H}_{2} \mathrm{O}$. Then, inflate cuff of the endotracheal tube to the minimum pressure required to prevent air leak at a pressure of $20 \mathrm{~cm} \mathrm{H} 2 \mathrm{O}$, listening over the trachea to identify air leak if present.

NOTE: This is important to prevent mucosal ischemia during intermittent positive pressure ventilation.

3. Normoxia and normocarbia are maintained during anesthesia.

8. Start administration of $2 \%$ isoflurane in $50 \%$ oxygen $/ 50 \%$ air. Titrate oxygen to maintain $\mathrm{PaO}_{2}$ of $90-100 \mathrm{mmHg}$. Continue for $3 \mathrm{~h}$ (or desired experimental duration).

9. Apply ophthalmic ointment on eyes to prevent dryness for the duration of anesthesia.

2. Begin femoral artery catheterization after initiation of $2 \%$ isoflurane.

1. Administer broad-spectrum antibiotics pre-incision (cefazolin, $25 \mathrm{mg} / \mathrm{kg}$ ) via the peripheral intravenous line to prevent surgical site infection.

2. Sterilize both groins using tinted chlorhexidine to ensure proper sterile field, and place an appropriate sterile drape (Figure 3B). At a minimum, staff participating in survival surgery should wear a surgical cap, mask, sterile gloves, and eye protection.

3. Palpate the femoral pulse at the inguinal crease using index and middle fingers.

4. Make a superficial, $1.5 \mathrm{~cm}$, craniocaudal incision using a scalpel (Figure $3 \mathrm{C}$ ).

5. Dissect between the two heads of the gracilis muscle using a blunt instrument, such as surgical hemostat or blunt-tipped scissors (Figure 3D).

NOTE: The femoral neurovascular bundle is typically found between and just deep to these two muscles. (Figure 4A).

6. Using vascular loops or silk ties, isolate the artery at the proximal and distal end. Use the loop to pull the artery up to the level of the skin distally (Figure 4B).

7. While placing traction on the proximal tie sufficient to interrupt blood flow, make a small arteriotomy with a pair of tenotomy scissors.

1. Take caution to not transect the artery. A small arteriotomy is sufficient. Alternatively, use a needle-and-wire approach to access the artery (Figure 4C). 
2. Gentle traction on the proximal tie will prevent excessive blood loss at any point during this portion of the procedure. If using the needle-and wire approach, pass the guidewire (supplied with the kit or obtained separately) through the needle and into the artery up to $5 \mathrm{~cm}$.

3. Take caution to not advance the wire further as it can cause ventricular ectopy. Withdraw the wire immediately $1-2 \mathrm{~cm}$ if this occurs.

8. Remove the needle from the vessel, taking care to leave the guide wire in the vessel. Gently pass the catheter over the wire and into the vessel (Figure 4D).

1. Use a 3-French, 8 centimeter catheter for femoral artery catheterization. Expect mild resistance when the tip of the catheter first enters the superficial wall of the vessel.

9. If using the arteriotomy approach, advance the catheter or polyethylene tubing directly into the vessel. Blood return should be observed immediately.

10. Immediately attach the catheter attached to a pressure transducer. Flush the catheter with normal saline to maintain catheter patency.

1. Place sutures to secure the catheter in place. Cover the incision with sterile gauze to prevent contamination. Utilize a percutaneous approach to femoral artery catheterization.

NOTE: Ensure that the ultrasound is performed by an experienced technician.

3. Intra-operative conditions and monitoring:

1. Actively warm the piglet with a forced-air warming device and continuously monitor rectal temperature (Figure $3 \mathbf{A}$ ).

2. Infuse dextrose-containing, isotonic fluid ( $5 \%$ dextrose in Ringer's lactate or normal saline) at maintenance rate (4 times the piglet's weight in kilograms, $\mathrm{mL} / \mathrm{hr}$ ).

3. Monitor vital signs for perturbations (hypotension, arrhythmia, hypo/hyperthermia, hypoxia)

NOTE: Normal vital sign ranges and corresponding suggested management in the case of abnormalities are summarized in Table 1.

4. Using a commercially available blood analysis system, measure arterial blood gases (arterial $\mathrm{pH}, \mathrm{pCO}_{2}, \mathrm{pO}_{2}$ ), electrolytes (bicarbonate, base excess/deficit, sodium, potassium, ionized calcium), hemoglobin, and glucose at least hourly during the experimental period. Draw arterial blood sample from the femoral artery catheter.

NOTE: Normal acid-base and electrolyte values along with recommendations for correction if abnormalities are seen are summarized in Table 1.

4. After $\mathbf{3} \mathbf{h}$ of isoflurane exposure, remove the femoral artery catheter.

1. Carefully tie down the proximal vascular silk to permanently occlude the femoral artery to prevent bleeding. Alternatively, use a vascular clip.

2. Ensure complete hemostasis before closing the incision. Irrigate the incision with $10-20 \mathrm{~mL}$ of sterile saline solution to help prevent infection.

5. At the conclusion of the experiment, close the skin incision with simple, interrupted sutures using a 3-0 non-absorbable suture material.

1. Infiltrate the wound using $0.5-1 \mathrm{~mL} / \mathrm{kg}$ of $0.25 \%$ bupivacaine with $1: 200,000$ epinephrine for pain control. Coat the incision with a sterile, surgical skin adhesive.

NOTE: No dressing is required.

6. Discontinue the anesthetic and allow the piglet to awaken.

7. Remove the endotracheal tube upon signs of awakening (opening of the eyes, attempts to stand, kicking, and opening and closing of the mouth), with signs of stable hemodynamics, adequate oxygenation, and adequate ventilation.

8. After extubation, supply supplemental oxygen via face cone until adequacy of oxygenation and ventilation are assured.

9. Administer buprenorphine $0.05 \mathrm{mg} / \mathrm{kg}$ subcutaneously for additional pain control. Alternatively, transdermal fentanyl can be used.

10. When appropriate, return the piglet to its home cage. Do not leave the piglet unattended until it has regained sufficient consciousness to maintain sternal recumbency. Do not return an animal to a cage with other animals until it has fully recovered from anesthesia.

1. Actively warm the private home cage with a warming light.

2. Closely monitor the animal after anesthesia by trained veterinary or research staff. Provide the piglet milk replacer.

11. Allow the piglets to recover for 48-72 $\mathrm{h}$, depending on experimental goals.

1. Inject piglets with buprenorphine subcutaneously at an appropriate dose, every three hours as needed to ensure pain control, at the discretion of animal care staff experienced to control post-surgical discomfort in animals.

2. Monitor the piglets every hour for the first $6 \mathrm{~h}$ after surgery and every $4 \mathrm{~h}$ thereafter. Perform animal sacrifice and tissue procurement in identical fashion to control animals, described above. 


\section{Representative Results}

Forty piglets were studied (18 isoflurane, 22 control). Study procedures were well tolerated by all animals. All piglets studied were male. There was no significant difference between the groups with respect to age or weight (Table 2, Figure 5). Average laboratory values during experimentation in the isoflurane group are given in Table 3. These values demonstrate that the experimental protocol possesses internal consistency and reproducibility, as we had multiple technicians performing the surgery over the course of two years. Unsaid by these numbers are the many adjustments and corrections we had to perform during the surgeries to maintain physiologic hemostasis. $\mathrm{CO}_{2}$ retention, low core body temperature, and hypoglycemia are some of the many confounders that we averted through comprehensive monitoring and adjustment as necessary.

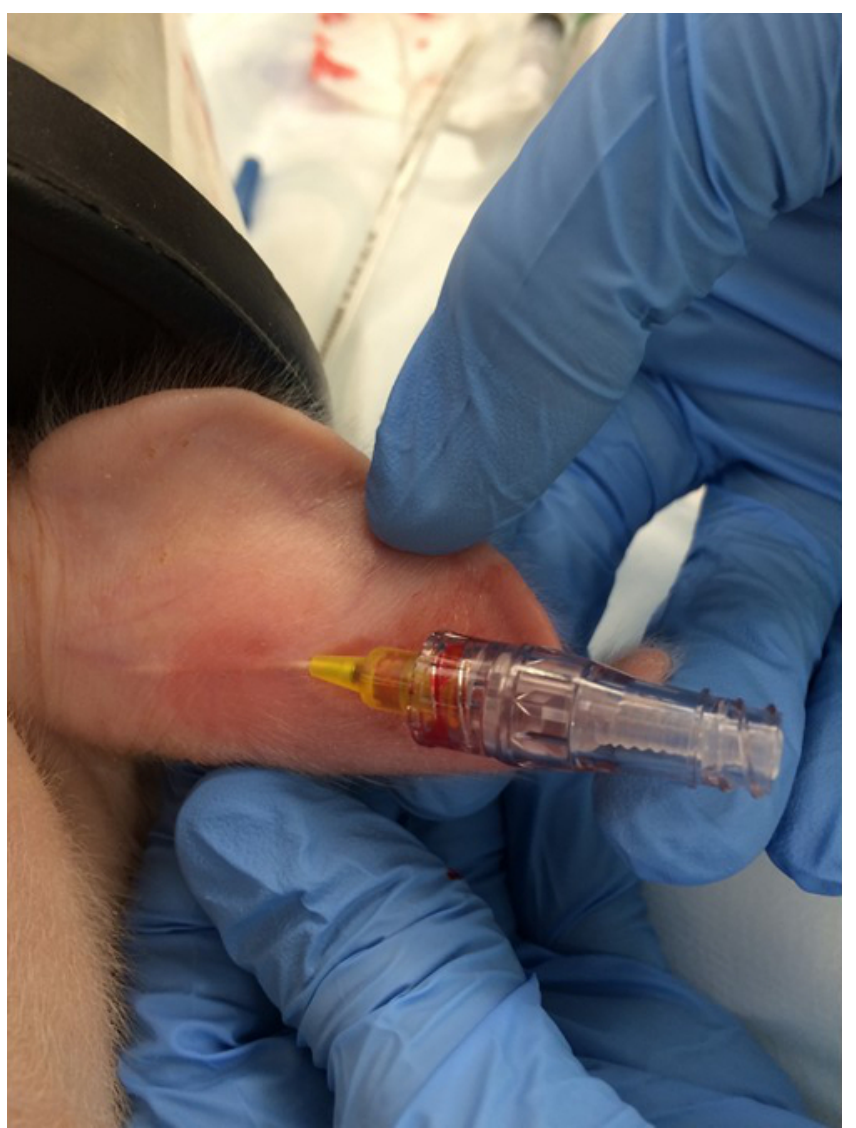

Figure 1:. Placement of the Peripheral Intravenous Line. A $24 \mathrm{G}$ intravenous catheter is placed in the marginal ear vein. Please click here to view a larger version of this figure. 


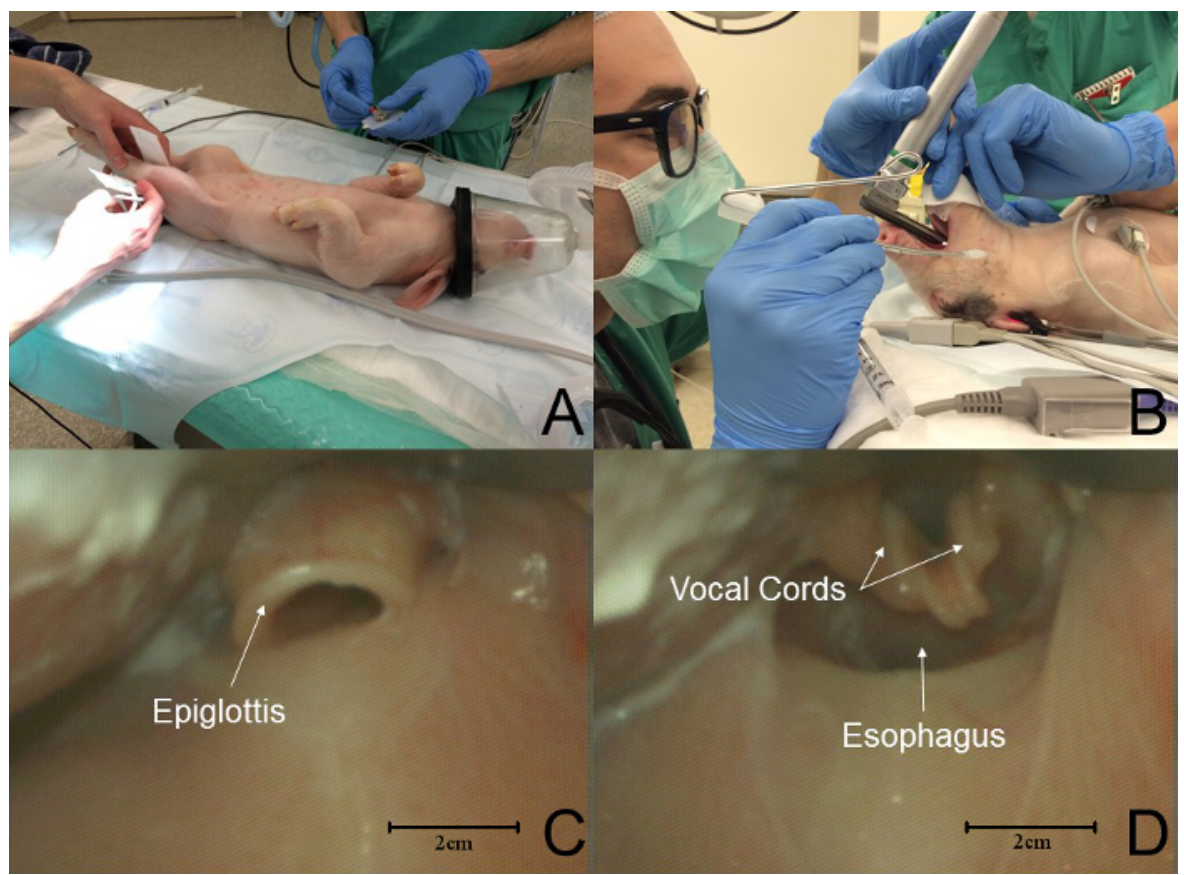

Figure 2: Sequence of Events in Piglet Intubation. (A) Piglets are placed in the lateral recumbent position for intubation. Standard monitors are placed. (B) An assistant displaced the tongue from the oral cavity while the laryngoscopist performs laryngoscopy. (C) The epiglottis is morphologically similar to that of a human. In this graphic, the tip of the blade is in the vallecula. (D) Porcine laryngeal anatomy is distinctly different from that of humans; the vocal cords are several millimeters deep to the laryngeal inlet. In this graphic, the tip of the blade has displaced the epiglottis, exposing the larynx. Please click here to view a larger version of this figure.

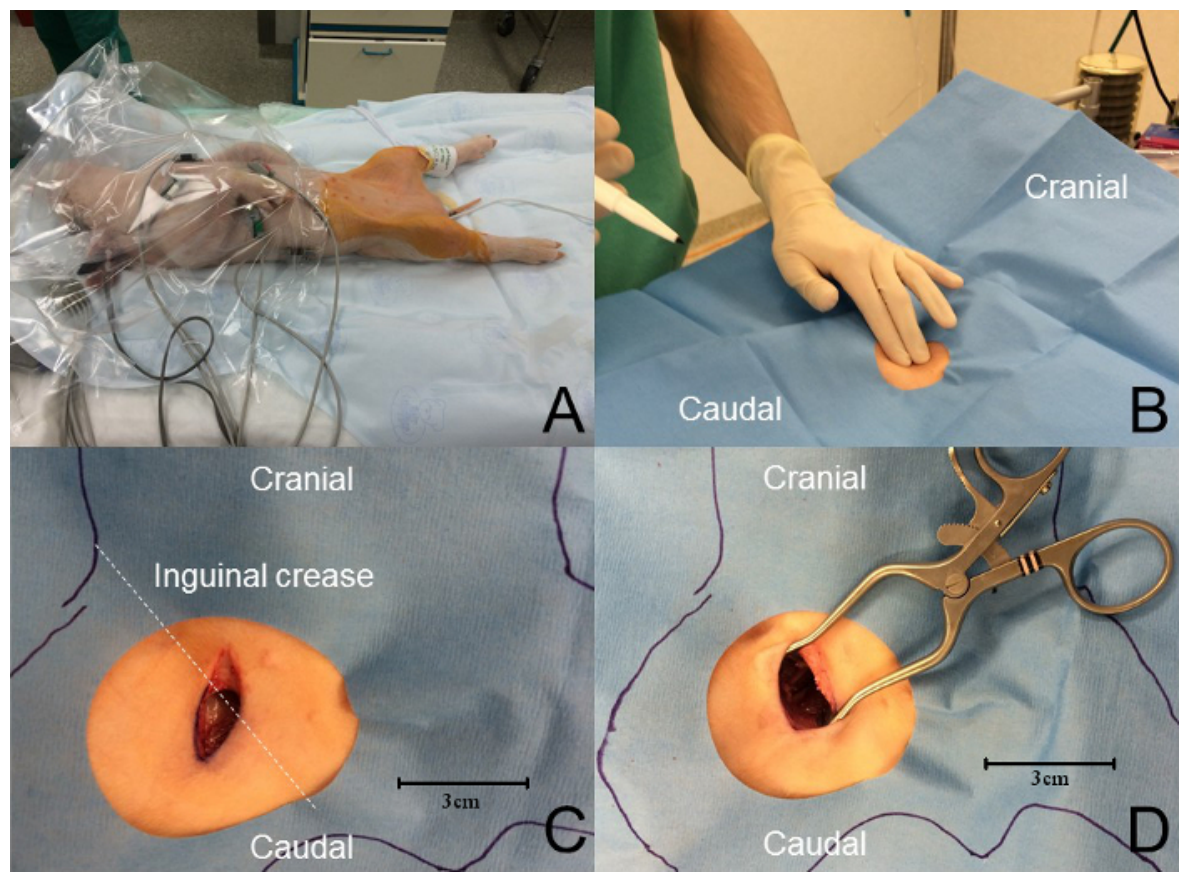

Figure 3: Femoral Artery Approach. (A) The animal temperature is maintained using a forced-air warming device. Monitoring is used throughout the procedure. A wide sterile field is prepared with tinted chlorhexidine and the animal is covered with a fenestrated sterile drape. (B) Palpation at the inguinal crease reveals the femoral pulse. (C) A craniocaudal skin incision, roughly perpendicular to the inguinal crease and approximately $1.5 \mathrm{~cm}$ in length, is performed overlying the femoral pulse. (D) A blunt dissection is achieved to expose the femoral neurovascular bundle. Please click here to view a larger version of this figure. 


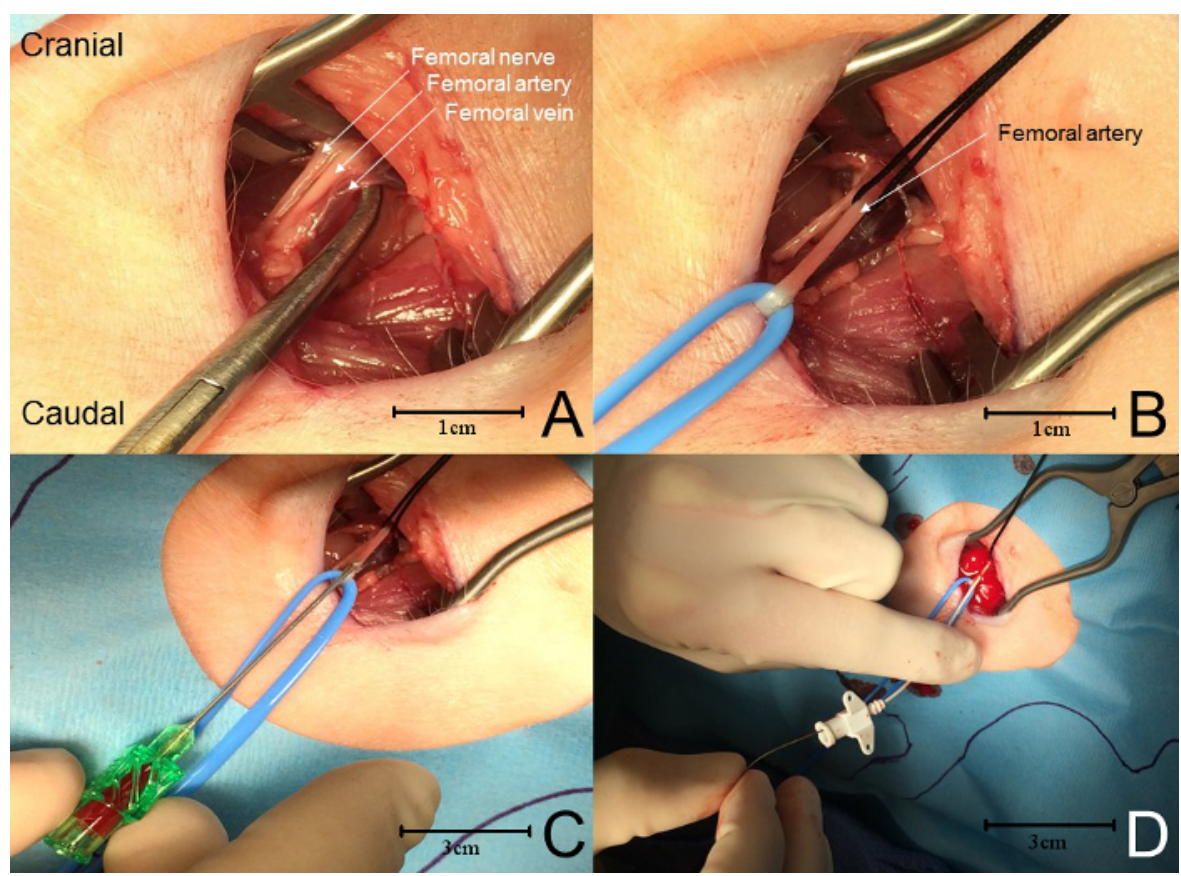

Figure 4: Femoral Artery Cannulation. (A) From lateral to medial, the neurovascular bundle contains the femoral nerve, artery, and vein. (B) The femoral artery is isolated using vessel loops and/or suture. Sustained tension on the proximal tie prevents excessive blood loss while the artery is punctured (see whitening of the vessel). (C) A needle is used to puncture the femoral artery, avoiding perforation of the posterior wall of the artery. When blood returns, a guidewire is advanced into the artery through the needle. (D) The needle is removed and the catheter is advanced over the guidewire into the femoral artery. Please click here to view a larger version of this figure.

\section{Age (days)}

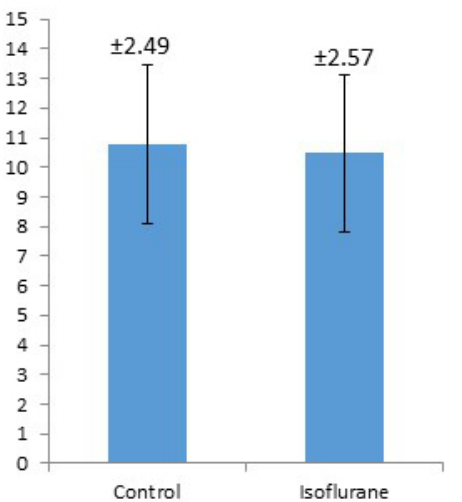

Weight (kg)

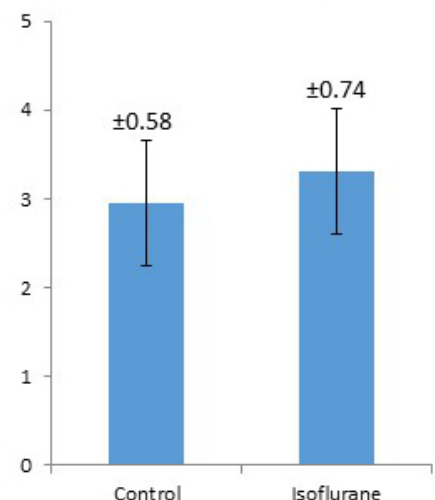

Figure 5: Comparison of Average Weight and Age of Control and Isoflurane-treated Piglets. The error bars represent $+/-1$ standard deviation, with the standard deviation value noted above each error bar. 


\begin{tabular}{|c|c|c|}
\hline Parameter & Normal Range & Correction \\
\hline pH & $7.35-7.45$ & Acdosis/alkalosis is usually respiratery; correct vinth wentlator settings \\
\hline $\mathrm{PCO}_{2}(\mathrm{mmH})$ & 35.45 & Hyper/hypocarbla usually due to ventllator support, correct wit th ventilator settings \\
\hline $\mathrm{pO}_{2}(\mathrm{mmHz})$ & $\begin{array}{c}90-100 \\
{\left[50 \% 6 \mathrm{FiO}_{2}\right]}\end{array}$ & 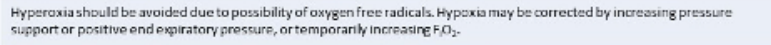 \\
\hline $\mathrm{HCO}_{3}(\mathrm{mmol} / \mathrm{L})$ & $22-33$ & Perturtations are typically respiratory in nature. Correct ventistor settiress sactordingly \\
\hline Sodlum (mmol/L) & $129-143$ & Increase or cecrease isotonic fluid infusion rate \\
\hline Potassium (mmol/L) & 3.9-4.1 & Hyper/hypokalemia are rarely seen intraoperatwely, muls perturbatiors do not need correction \\
\hline lonized Calcium (mmol/L) & 1.1-1.6 & For hypocalcemia, consider calcium gluconate $30 \mathrm{~m} / / \mathrm{kg} /$ dose titrated to laboratory parameters \\
\hline Glucose (mg/dL) & $100-200$ & Increase or decrease dextrose contaning, isotonk fluw infusicn rate \\
\hline Hemoglobin (e/dt) & 7-10 & $\begin{array}{l}\text { In the absence of acute blood loss during surgery, anemia is usually oue to a dilutional effect from intravenous fluid } \\
\text { administration. No therapy is required. }\end{array}$ \\
\hline $\begin{array}{l}\text { Systolic Blood Pressure } \\
\text { (mmHE) }\end{array}$ & $65-95$ & 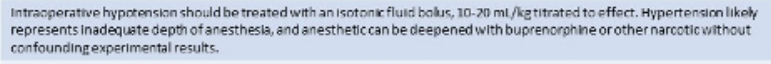 \\
\hline $\begin{array}{l}\text { Diastolic Blood Pressure } \\
\text { (mmHE) }\end{array}$ & $35-55$ & $\begin{array}{l}\text { Hypertansion likely represents inadequate dopth of anesthesia, and anesthetic can be doepened with bupromorphine or other } \\
\text { narcotic without confounding expermental results. }\end{array}$ \\
\hline Heart Rate (beats per minute) & $120-200$ & 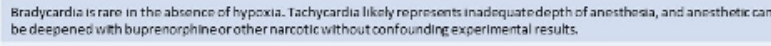 \\
\hline Oxygen Saturation (\%) & 90-100 & $\begin{array}{l}\text { Most piglets will require pressire support i frespiration is spontaneaus under anesthesia. Increasine pressure support or } \\
\text { positive-end expiratory pressureotten corrects hypossa in anesthetized piglets. }\end{array}$ \\
\hline $\begin{array}{l}\text { Respiratory Rate (breaths per } \\
\text { minute) }\end{array}$ & $30-60$ & 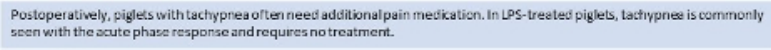 \\
\hline $\begin{array}{l}\text { End-Tidal Carbon Dioxide } \\
\text { (mmHg) }\end{array}$ & $35-45$ & Titrate ventilator support as inclicated. \\
\hline Core Temperature $\left[{ }^{\circ} \mathrm{C}\right]$ & $101-103$ & $\begin{array}{l}\text { Hypothermia can be avoided by housing piglets intemperature-controled cages and by maintaining activewarming during } \\
\text { surgery. Hyperthermia may be a sign of malignant hyperthernia and should be taken serizusly. }\end{array}$ \\
\hline
\end{tabular}

Table 1: Summary of Normal Piglet Vital Signs, Arterial Blood Gas, and Serum Electrolyte Values with Suggested Correction Methods. Please click here to view a larger version of this table.

\begin{tabular}{|l|c|l|l|}
\hline & \multicolumn{1}{|c|}{ Control $(\mathrm{n}=\mathbf{2 2})$} & \multicolumn{1}{|c|}{ Isoflurane $(\mathrm{n}=\mathbf{1 8})$} & \multicolumn{1}{c|}{ P value } \\
\hline Average Age (days, \pm SD) & $10.77 \pm 2.49$ & $10.5 \pm 2.57$ & 0.708 \\
\hline Average Weight $(\mathrm{kg}, \pm$ SD) & $2.95 \pm 0.577$ & $3.31 \pm 0.737$ & 0.091
\end{tabular}

Table 2: Summary of Average Weight and Age of Control vs Isoflurane-treated Piglets. An unpaired two tailed T test was performed, showing no significant difference between the two groups. Please click here to view a larger version of this table.

\begin{tabular}{|l|c|c|}
\hline \multicolumn{2}{|l|}{ Table 3. Average Arterial Blood Gases and Serum Electrolytes of Isoflurane Piglets } \\
\hline & Mean \pm SD & Median \\
\hline $\mathrm{pH}$ & $7.44 \pm 0.05$ & 7.44 \\
\hline $\mathrm{pCO}_{2}(\mathrm{mmHg})$ & $41.7 \pm 4.93$ & 41.3 \\
\hline $\mathrm{pO}_{2}(\mathrm{mmHg})$ & $231 \pm 46$ & 230 \\
\hline $\mathrm{HCO}_{3}(\mathrm{mmol} / \mathrm{L})$ & $28.8 \pm 3.16$ & 28.3 \\
\hline Sodium $(\mathrm{mmol} / \mathrm{L})$ & $137 \pm 5.12$ & 136 \\
\hline Potassium (mmol/L) & $4.92 \pm 0.88$ & 4.80 \\
\hline lonized Calcium (mmol/L) & $1.51 \pm 0.11$ & 1.50 \\
\hline Base Excess & $4.51 \pm 3.35$ & 4.67 \\
\hline Hemoglobin (g/dL) & $5.44 \pm 3.28$ & 4.45 \\
\hline Glucose (mg/dL) & $173 \pm 43.7$ & 159 \\
\hline
\end{tabular}

Table 3: Average Vital Signs and Lab Values of Isoflurane-treated Animals. During the course of the surgeries, deviations of the vital signs and lab values from the normal ranges are promptly corrected. Please click here to view a larger version of this table.

\section{Discussion}

\section{Critical Protocol Steps/Troubleshooting}

As the experiment begins, monitoring of non-invasive vital signs should begin with induction. Blood pressure, heart rate, oxygen saturation, and rectal temperature can be readily obtained and monitored. The piglet should be under an air-warming device to maintain adequate core body temperature, as these animals can become hypothermic quickly under general anesthesia. Prompt placement of a peripheral intravenous catheter allows for treatment of emergencies if they arise during induction. It is important to continuously monitor the piglet, noninvasively or invasively, throughout both the isoflurane procedure and the sacrifice procedure. The piglet can experience arterial oxygen desaturation very rapidly during multiple steps throughout the protocol, especially during airway management and intubation. We use $8 \%$ sevoflurane to induce anesthesia in order to replicate human pediatric practice and to speed induction. However, $5 \%$ isoflurane has been used successfully and is appropriate. Given the differences in anatomy and a predisposition for laryngospasm, the piglet can be difficult to intubate. If the piglet starts to desaturate during the induction and/or airway management, $100 \%$ oxygen and sevoflurane should immediately be administered via face cone 
in order to reestablish a safe oxygen saturation level and an adequate depth of anesthesia. Keep in mind that while the plane of anesthesia must be deep enough to allow intubation, excessive anesthesia may lead to apnea. Continuous vigilance with respect to the animal's ventilation and oxygenation is required, with titration of inhaled anesthetic accordingly. Intubation may then be reattempted once oxygenation is restored and adequate anesthesia has been achieved. Positive pressure ventilation via the face cone may be attempted, but is usually unsuccessful. If laryngospasm occurs, application of lidocaine solution directly to the vocal cords is indicated to allow tracheal intubation.

Emergency medications should always be available and should be administered as needed during the critical portions of the protocol to correct physiologic disturbances. While a thorough discussion of anesthetic and emergency drug use in piglets is outside the scope of this manuscript, Swindle's "Swine in the Laboratory: Surgery, Anesthesia, Imaging, and Experimental Techniques" is an excellent resource.

Similarly, the piglet may begin to desaturate rapidly during the sacrifice, after opening the chest cavity during the midline sternotomy. The operator should work quickly but safely to expose the heart and insert the angiocatheter to start the cold PBS. A thorough cold PBS perfusion (and rapid fixation with PFA, if indicated) is needed to prevent ischemic damage to the brain.

Once the piglet has been intubated, respiratory rate and end-tidal carbon dioxide tracking begins (Table 1). Stabilize the piglet's end-tidal oxygenation and ventilation by titrating ventilator support while maintaining adequate anesthesia. We use mechanical ventilation to mimic what is used in humans as closely as possible. Hyperoxia should be avoided to minimize the chance of oxidative stress.

The isoflurane piglets undergo a femoral artery cannulation for 2 reasons: to continuously monitor arterial blood pressure; and to sample arterial blood for assessment of acid-base status, blood gases, and electrolytes throughout the procedure. Cannulation of the femoral artery can be challenging. Please see the video for full details. For survival experiments, this procedure should be done in a sterile operating environment under sterile conditions. After cannulation of the femoral artery, begin hourly monitoring of arterial blood gas and serum electrolytes, correcting as necessary to maintain homeostasis (Table 1). The piglet should receive continuous dextrose-containing isotonic fluid to maintain adequate blood glucose. Throughout the experiment, the animal should be continuously monitored for normothermia, and forced-air warming should be provided as needed. It is equally important to avoid hypothermia and hyperthermia.

While this protocol provides one hemisphere of "fresh" brain and one hemisphere of fixed neural tissue, this can be easily adapted to accommodate alternative study designs. Additional samples can be collected from the piglet as well. CSF can be obtained after anesthetizing the piglet, with or without fluoroscopy guidance. Blood may also be collected from the piglet at various stages of the protocol, including the from the femoral artery catheter, as well as directly from the left ventricle via the angiocatheter immediately prior to perfusion. The convalescence period may also be lengthened or shortened, for examination of the chronic or acute response, respectively.

\section{Limitations of the Technique}

This protocol and model are technically challenging. A skilled investigator and a fully supplied operating suite are required, particularly for survival experiments. The investigator (and assistant, for certain portions of the protocol) must be comfortable with both the surgical and anesthetic components of this protocol, which may require training and experience to master. Other limitations include the expense of the piglets relative to rodent models, though the piglet model is far less cost-prohibitive than non-human primates. While the cost of piglets will vary based upon the region and farm from which animals are obtained, one can expect the per-animal cost to be less than $\$ 500$, whereas non-human primates can be thousands of dollars per animal. In our experience, the average cost per animal is typically about $\$ 200$.

Finally, as it is the purpose of the piglet model to mimic the developing human brain, only neonatal piglets should be used. The central nervous system is most vulnerable during the period of rapid growth, and in piglets, this period extends from six weeks prior to birth to five weeks after birth $^{8}$. Using older piglets more distant from their farrowing date carry the risk of weakening the clinical relevance of the piglet model. While significant controversy exists with regards to the "equivalency" of piglet brain development to that of a human neonate, there are striking similarities when early postnatal brain development between humans and pigs is compared.At birth, the brains of humans and pigs are $27 \%$ and $25 \%$ of adult weight, respectively. ${ }^{14}$ Based upon the work of Johnson and colleagues, we can infer that one piglet week is roughly equivalent to one human month. ${ }^{9}$ These results, based upon whole-brain volume data, have been validated by the work of Workman and colleagues. ${ }^{28}$ We selected 7-14 day old piglets in order to approximate a human of 1-2 month of age. However, it may be prudent to use younger animals (1-5 days old) if the experimental goal is to mimic the zenith of the piglet brain growth spurt. This is feasible, as piglets can be weaned at birth. Our use of the piglet model will adapt as new data become available with respect to the parallels between human and pig postnatal brain development.

\section{Significance of the Technique with Respect to Alternative/Existing Methods}

The piglet possesses striking similarities to human neonates, including critical parallels in brain development and pathophysiologic responses. It is therefore a clinically relevant mammalian model and the proof-of-concept study indicates that the piglet is a suitable model for the study of anesthetic neurotoxicity ${ }^{29,30}$. It can also be easily adapted to other types of developmental neuroscience research. The model is designed to investigate, with scientific authority, the extent and mechanism of AIDN minimizing concerns that confounders, such as hypoxia or hypercarbia, are causing neurologic damage that could be misinterpreted as anesthesia-induced. To accomplish this, the piglet is treated with the same perioperative surgical and anesthetic conditions and monitoring experienced by pediatric patients.

\section{Future Directions and Applications After Mastering the Technique}

Moving forward, piglets are also very amenable to neurocognitive testing ${ }^{17}$. This attribute will allow for complex, comprehensive evaluation of neurocognitive outcome after anesthetic exposure in future experiments. It should also be emphasized that in the clinical setting, children most often undergo anesthesia for a physiologically stressful procedure (surgery). The interactions between anesthesia and post-surgical inflammation as well as the resultant neuronal injury and/or toxicity (as seen in rodents and primates) deserve further exploration and significant consideration. The neonatal piglet provides a unique, clinically relevant baseline model for the effects of anesthetics on the developing brain without the confounding influence of surgery (mimicking common clinical scenarios in children). The impact of different types of surgery or other confounders (ischemia, brain injury, genetic predisposition, etc.) can now be reliably tested using this model. 
In laboratory, we plan to use multiple electrophysiological and electrochemical methods to further investigate mechanisms of anesthesia and AIDN in intact neural circuits. These techniques include in vivo measurement of neurotransmitter activity, whole-cell patch-clamp recordings, neuroimaging, and neurophysiological investigations in brain slices. With respect to neuroscience in the immature brain, piglets are more relevant to humans than murine models with very few of the drawbacks present with non-human primates. With further development, piglets may be the ideal model for human developmental neuroscience research.

\section{Disclosures}

The authors declare that they have no competing interests.

\section{Acknowledgements}

The authors would like to acknowledge the contributions of the Ohio State University Laboratory Animal Resource Center (ULAR).

\section{References}

1. Buie, V. C., Owings, M. F., DeFrances, C. J., \& Golosinskiy, A. National hospital discharge survey: 2006 annual summary. Vital Health Stat. 13. (168) 1-79 (2010).

2. Hays, S. R., \& Deshpande, J. K. Newly postulated neurodevelopmental risks of pediatric anesthesia. Curr Neurol Neurosci Rep. 11, 205-210 (2011).

3. Disma, N., Mondardini, M. C., Terrando, N., Absalom, A. R., \& Bilotta, F. A systematic review of methodology applied during preclinical anesthetic neurotoxicity studies: important issues and lessons relevant to the design of future clinical research. Paediatr Anaesth. 26, 6-36 (2016).

4. Loepke, A. W., \& Vutskits, L. What lessons for clinical practice can be learned from systematic reviews of animal studies? The case of anesthetic neurotoxicity. Paediatr Anaesth. 26, 4-5 (2016).

5. Cherubini, E., Rovira, C., Gaiarsa, J. L., Corradetti, R., \& Ben Ari, Y. GABA mediated excitation in immature rat CA3 hippocampal neurons. International Journal of Developmental Neuroscience. 8, 481-490 (1990).

6. Kaindl, A. M. et al. Brief alteration of NMDA or GABAA receptor-mediated neurotransmission has long term effects on the developing cerebral cortex. Mol Cell Proteomics. 7, 2293-2310 (2008).

7. Glauser, E. M. Advantages of piglets as experimental animals in pediatric research. Exp Med Surg. 24, 181-190 (1966).

8. Dickerson, J., \& Dobbing, J. Prenatal and postnatal growth and development of the central nervous system of the pig. Proceedings of the Royal Society of London B: Biological Sciences. 166, 384-395 (1967).

9. Conrad, M. S., \& Johnson, R. W. The domestic piglet: an important model for investigating the neurodevelopmental consequences of early life insults. Annu Rev Anim Biosci. 3, 245-264 (2015).

10. Nielsen, K. B. et al. Reelin expression during embryonic development of the pig brain. BMC Neuroscience. 11, 75 (2010).

11. Niblock, M. M. et al. Comparative anatomical assessment of the piglet as a model for the devleoping human medullary serotonergic system. Brain Res Brain Res Rev. Vol. 50, Netherlands, 169-183, (2005).

12. van Straaten, H. W., Peeters, M. C., Hekking, J. W., \& van der Lende, T. Neurulation in the pig embryo. Anat Embryol (Berl). 202, 75-84, (2000).

13. Goureau, A. et al. Conserved synteny and gene order difference between human chromosome 12 and pig chromosome 5 . Cytogenetics and Cell Genetics. 94, 49-54 (2001).

14. Dobbing, J., \& Sands, J. Comparative aspects of the brain growth spurt. Early Human Development. 3, 79-83 (1979).

15. Istaphanous, G. K. et al. Characterization and quantification of isoflurane-induced developmental apoptotic cell death in mouse cerebral cortex. Anesth Analg. 116, 845-854 (2013).

16. Loepke, A. W. et al. The effects of neonatal isoflurane exposure in mice on brain cell viability, adult behavior, learning, and memory. Anesth Analg. 108, 90-104 (2009).

17. Sullivan, S. et al. Improved behavior, motor, and cognition assessment in neonatal piglets. J Neurotrauma. Vol. 30, 1770-1779 (2013).

18. Gieling, E. T., Nordquist, R. E., \& van der Staay, F. J. Assessing learning and memory in pigs. Anim Cogn. 14, 151-173 (2011).

19. Broom, D. M., Sena, H., \& Moynihan, K. L. Pigs learn what a mirror image represents and use it to obtain information. Animal Behaviour. 78, 1037-1041 (2009).

20. Martin, L. J., Spicer, D. M., Lewis, M. H., Gluck, J. P., \& Cork, L. C. Social deprivation of infant rhesus monkeys alters the chemoarchitecture of the brain: I. Subcortical regions. The Journal of Neuroscience. 11, 3344-3358 (1991).

21. Rizzi, S., Ori, C., \& Jevtovic-Todorovic, V. Timing versus duration: determinants of anesthesia-induced developmental apoptosis in the young mammalian brain. Annals of the New York Academy of Sciences. 1199, 43-51 (2010).

22. Brambrink, A. M. et al. Isoflurane-induced neuroapoptosis in the neonatal rhesus macaque brain. Anesthesiology. 112, 834-841 (2010).

23. Elmore, M. R. et al. Respiratory viral infection in neonatal piglets causes marked microglia activation in the hippocampus and deficits in spatial learning. J Neurosci. 34, 2120-2129 (2014).

24. Alonso-Alconada, D. et al. Brain cell death is reduced with cooling by 3.5 degrees $C$ to 5 degrees $C$ but increased with cooling by 8.5 degrees C in a piglet asphyxia model. Stroke. 46, 275-278 (2015).

25. Costine, B. A. et al. The subventricular zone in the immature piglet brain: anatomy and exodus of neuroblasts into white matter after traumatic brain injury. Developmental Neuroscience. Vol. 37, Switzerland, 115-130 (2015).

26. Holtzman, D. et al. In vivo phosphocreatine and ATP in piglet cerebral gray and white matter during seizures. Brain Research. 783, 19-27 (1998).

27. Swindle, M. M. Swine in the Laboratory. 2nd edn. CRC Press. (2007).

28. Workman, A. D., Charvet, C. J., Clancy, B., Darlington, R. B., \& Finlay, B. L. Modeling transformations of neurodevelopmental sequences across mammalian species. J Neurosci. 33, 7368-7383 (2013).

29. Lunney, J. K. Advances in swine biomedical model genomics. International Journal of Biological Sciences. 3, 179-184, (2007). 
30. Nemzek, J. A., Hugunin, K. M., \& Opp, M. R. Modeling sepsis in the laboratory: merging sound science with animal well-being. Comparative Medicine. 58, 120-128 (2008). 